\title{
Mejores prácticas en preparación de alimentos en micro y pequeña empresa
}

José Remberto Miranda-Mejía'

Recibido: 14/03/2014 - Aceptado: 30/05/2014

\section{Resumen}

El estudio evaluó los conocimientos sobre buenas prácticas en preparación de alimentos. Las buenas prácticas comprenden el manejo de temperaturas de los alimentos, la limpieza e higienización para lograr mantener la inocuidad en los alimentos. Se realizó un estudio descriptivo de corte transversal, durante el período del 14 al 30 de mayo del 2013. Se evaluaron nueve diferentes tipos de alimentos, de los cuales la mayoría se encuentra contaminado de bacterias, entre las que se menciona la Escherichia coli. Existe la probabilidad de que no todos los alimentos estén contaminados en las instalaciones en estudio, ya que el muestreo no fue aleatorio, por tal razón no puede generalizarse la contaminación de los alimentos de la zona en estudio.

\section{Palabras clave}

Higienización, inocuidad, elaboración de alimentos, temperaturas de alimentos.

\section{Abstract}

The study assessed the existing knowledge about good practices in food preparation. Such good practices include food temperature management, plus the cleaning and sanitation processes in order to maintain safety in food. A descriptive cross-sectional study was conducted during the period comprised between May 14 and May 30, 2013. Nine different types of food where evaluated, most of which were contaminated by bacterium, including Escherichia coli. There is a probability that not all foods in the site under study are contaminated, since the sample was not random, therefore, food contamination cannot be generalized in the area of study.

\section{Keywords}

Sanitation, safety, food processing and food temperatures.

1 José Remberto Miranda Mejía es ingeniero industrial. Actualmente trabaja como investigador de la Universidad Tecnológica de El Salvador y docente en Ingeniería de Métodos. jose.miranda@utec.edu.sv 


\section{Introducción}

Los manipuladores de alimentos son aquellas personas que, por su actividad laboral, tienen contacto directo con los alimentos durante su preparación y los pasos que son parte del proceso, como la fabricación, transformación, elaboración, envasado, almacenamiento y transporte. El deterioro de los alimentos ocasiona pérdidas, es costoso y puede influir negativamente en el comercio y en la confianza de los consumidores. Está demostrada la relación existente entre una inadecuada manipulación de los alimentos y la producción de enfermedades trasmitidas a través de estos. Las medidas más eficaces en la prevención de estas enfermedades son las higiénicas, ya que en la mayoría de los casos es el manipulador el que interviene como vehículo de transmisión, por actuaciones incorrectas, en la contaminación de los alimentos (Fundación vasca para la seguridad alimentaria, 2013).

Todas las personas tienen derecho a esperar que los alimentos que comen sean inocuos y aptos para el consumo. Las enfermedades de transmisión alimentaria y los daños provocados por los alimentos son desagradables $y$, a veces, ponen en riesgo la vida de muchas personas (OMS/FAO, 1969). Se ha calculado que cada año mueren 1.8 millones de personas como consecuencia de enfermedades diarreicas, cuya causa puede atribuirse en la mayoría de los casos a la ingesta de agua o alimentos contaminados. Una preparación adecuada de los alimentos puede prevenir la mayoría de las enfermedades de transmisión alimentaria (OMS, 2007). Las buenas prácticas de manipulación (BPM) son una herramienta básica para la seguridad alimentaria y representan los procedimientos mínimos exigidos en los mercados nacional e internacional en cuanto a higiene y manipulación de alimentos. Además engloba aspectos de diseño de instalaciones, equipos, control de operaciones e higiene del personal.

\section{Objetivo general}

Determinar la aplicación de las normas de buenas prácticas de manufactura en la elaboración de los alimentos en las instalaciones que venden comida en la zona centro de San salvador que forman la zona de estudio.

\section{Objetivos específicos}

Examinar los requisitos sanitarios que deben cumplir los establecimientos alimentarios en sus instalaciones y su funcionamiento. Identificar la presencia de agentes bacteriológicos en los alimentos ofrecidos por las micros y pequeñas empresas de comida de la zona en estudio. Evaluar los conocimientos sobre buenas prácticas en manipulación de alimentos de los manipuladores de comida en las instalaciones que están en la zona en estudio antes mencionada. Verificar la aplicación de las buenas prácticas en la preparación de comidas y bebidas.

\section{Marco teórico}

Mejores prácticas en la preparación de alimentos en micro y pequeña empresa.

\section{Elaboración de alimentos}

\section{Principios de higiene en los alimentos}

Los alimentos presentan microorganismos en su superficie o en su interior siempre. Estos pueden estar presentes en el interior de las estructuras del alimento donde pueden provocar zoonosis, enfermedades animales no transmisibles al hombre y enfermedades vegetales no transmisibles al hombre o en el exterior (exógenos, los que se incorporan al alimento durante su manipulación y procesado); y atendiendo a su relación con el consumidor, pueden ser agentes patógenos o alterantes. Los agentes endógenos o son inocuos (patógenos de plantas) o son eliminados en mataderos (animales enfermos) o durante el procesado (pasteurización) (OPS, 2013) (Red Tercer Milenio, 2012).

\section{Técnicas de las buenas prácticas de manipulación de alimentos}

Las materias primas deben ser almacenadas en condiciones apropiadas que aseguren la protección contra contaminantes. El depósito debe estar alejado de los productos terminados para impedir la contaminación cruzada. Además, deben tenerse en cuenta las condiciones óptimas de almacenamiento como temperatura, humedad, ventilación e iluminación.

Las materias primas utilizadas no deben contener parásitos, microorganismos o sustancias tóxicas, descompuestas o extrañas. Todas las materias primas deben ser inspeccionadas antes de utilizarlas. En caso necesario debe realizarse un ensayo de laboratorio (FAO/OMS, 2003). 
Estructura. El establecimiento no tiene que estar ubicado en zonas que se inunden, que contengan olores objetables, humo, polvo, gases, luz y radiación que pueden afectar la calidad del producto que elaboran.

Higiene. Todos los utensilios, los equipos y los edificios deben mantenerse en buen estado de higiene, de conservación y de funcionamiento. Para la limpieza y la desinfección es necesario utilizar productos que no tengan olor, ya que pueden producir contaminación además de enmascarar otros olores. Para organizar estas tareas, es recomendable aplicar los POES (Procedimientos Operativos Estandarizados de Saneamiento) que describen qué, cómo, cuándo y dónde limpiar y desinfectar, así como los registros y advertencias que deben llevarse a cabo (C.A.A., 2013).

La capacitación es de importancia fundamental para cualquier sistema de higiene de los alimentos. Una capacitación y/o instrucción y supervisión insuficientes sobre la higiene, de cualquier persona que intervenga en operaciones relacionadas con los alimentos, representa una posible amenaza para la inocuidad de los productos alimenticios y su aptitud para el consumo humano (Organización Panamericana de la Salud, 2013).

Debe controlarse el estado de salud y la aparición de posibles enfermedades contagiosas entre los manipuladores. Por esto, las personas que están en contacto con los alimentos deben someterse a exámenes médicos no solo previamente al ingreso, sino en forma periódica. Cualquier persona que perciba síntomas de enfermedad tiene que comunicarlo inmediatamente a su superior.

\section{Finalidad del análisis de alimentos}

En el análisis de alimentos se busca verificar si se cumple o no con los requerimientos establecidos de calidad e inocuidad con la finalidad de proteger a los consumidores. Por ejemplo: el porcentaje de grasa en un queso fresco, los grados brix en un jugo de frutas, la cuenta de bacterias coliformes totales en un helado de fresa, la presencia de Escherichia coli 0157:H7 en hamburguesas de res, etc. (FAO, 2013).

\section{Método}

Se realizó un estudio descriptivo de corte transversal, durante el período del 14 al 30 de mayo de 2013 (Hernández, Fernández y Baptista, 2010).
El total de micros y pequeñas empresas que elaboran alimentos y que colaboraron con el estudio fue de 25 en la zona centro de San Salvador que fue el área de estudio. Solo se tomaron en cuenta aquellos que poseían instalaciones propias o alquiladas; el requisito es que no sean ventas ambulantes. La participación fue voluntaria, mediante consentimiento informado. En el estudio se incluye los datos de las encuestas de los manipuladores. Al final se logró entrevistar a 25 manipuladores de comida y se evaluó igual número de empresas con una guía de observación de las condiciones higiénicas, la ventilación, la iluminación de las instalaciones donde se prepara y venden comida.

Las encuestas se realizaron al personal que trabaja en los establecimientos que venden comida en el centro de San Salvador que forman la zona de estudio. También se utilizó una guía de evaluación para locales.

\section{Muestreo de puntos críticos}

Se tomaron muestras en ciertos puntos críticos para verificar la calidad del producto. Este tipo de muestreo generalmente se utiliza cuando se quiere corroborar 0 saber si un producto en un sitio específico cumple o no una especificación de calidad.

Se tomaron muestras en diferentes puntos críticos determinados por el investigador para ser analizadas. En estos casos el criterio de la selección de los puntos críticos no es aleatorio. El investigador los determina considerando el riesgo de que en ese sitio haya una contaminación (FAO, 2013)

En el muestreo de análisis de presencia de microorganismos en un alimento, se consideró en el plan de muestreo que el tiempo que pasa entre la toma de la muestra y el análisis sea lo más breve posible y que las condiciones de conservación durante su transporte y almacenamiento no aumente 0 disminuya la cantidad de organismos (FAO, 2013).

\section{Encuesta para manipuladores de alimentos}

En el primer instrumento, al inicio, se busca conocer tres variables sociodemográficas (edad, nivel de estudio y municipio de residencia); escala nominal, con preguntas abiertas hacia el entrevistado. La parte siguiente es un cuestionario con algunas preguntas cerradas y otras abiertas (dicotómicas y policotómicas). Con este tipo de preguntas 
se espera recolectar datos sobre las incidencias que se dan durante el proceso de elaboración de alimentos. También, el primer instrumento tiene partes de un test muy similar al cuestionario donde puede marcar una opción. Para su elaboración se tomó en cuenta el Manual de manipulación de alimentos (OMS, 2007). Su validación fue de contenido.

\section{Ficha de evaluación para locales}

La encuesta para locales es un test sencillo y objetivo para evaluar la aptitud/no aptitud de los locales donde se preparan y consumen las comidas, utilizando una escala de intervalo que se responde marcando una de las opciones entre una lista. El test mide las condiciones de ventilación, iluminación, control de insectos, plagas, conservación de productos y materias primas, limpieza, desinfección, higiene personal y requisitos sanitarios. Las evaluaciones están basadas según Codex Alimentarius (Codex Alimentarius, 2003). La validación fue por juicio de expertos.

\section{Análisis de resultados bacteriológicos}

Los resultados de los análisis microbiológicos realizados para detectar la presencia de microorganismos se presentan de la siguiente forma: coliformes totales, fecales y Escherichia coli, Salmonella spp, Staphylococcus aureus en unidades formadoras de colonias (UFC/g) por $25 \mathrm{~g}$ de muestra analizada; se determina la presencia o ausencia de $25 \mathrm{~g}$ de alimento; también número más probable en $25 \mathrm{~g}$ de muestra (NMP/g), dependiendo de los límites especificados por la norma para cada alimento. El laboratorio que evaluó las muestras de comida está acreditado con la norma NSR ISO/IEC 17025:05, cumpliendo con el estándar para muestreo de alimentos (FAO, 2013).

Tabla 1

Resultados de análisis bacteriológicos en alimentos

\begin{tabular}{|c|c|c|c|c|c|c|}
\hline Alimento & Escherichia Coli & Salmonella & Staphylococcus aureus & Coliformes fecales & Coliformes totales & Conteo bacteriano total \\
\hline Arroz & $<3$ & Ausencia & $<10$ & -- & -- & -- \\
\hline Norma & $<3 \mathrm{NMP} / 100 \mathrm{ml}$ & Ausencia & $<10$ UFC $/ \mathrm{ml}$ & -- & -- & -- \\
\hline Pupusas & 240 & Ausencia & $<10$ & -- & -- & -- \\
\hline Norma & $<3 \mathrm{NMP} / 100 \mathrm{ml}$ & Ausencia & $<10$ UFC $/ / \mathrm{ml}$ & -- & -- & -- \\
\hline Tortilla & 93 & Ausencia & -- & -- & -- & 120,000 \\
\hline Norma & $<3 \mathrm{NMP} / 100 \mathrm{ml}$ & Ausencia & -- & -- & -- & -- \\
\hline Ensalada M. & 460 & Ausencia & -- & -- & $>1100$ & -- \\
\hline Norma & $<3 \mathrm{NMP} / 100 \mathrm{ml}$ & Ausencia & -- & -- & $93 \mathrm{NMP} / \mathrm{g}$ & -- \\
\hline Pollo & $<3$ & Ausencia & $<10$ & -- & -- & -- \\
\hline Norma & $<4 \mathrm{NMP} / 100 \mathrm{ml}$ & Ausencia & $<10$ UFC $/ \mathrm{ml}$ & -- & -- & -- \\
\hline Leche con $\mathrm{G}$. & 0.092 & Ausencia & $<1$ & 2.4 & 11 & -- \\
\hline Norma & Ausencia & Ausencia & Ausencia & Ausencia & $<10$ & 20,000 UFC* $/ \mathrm{ml}$ \\
\hline Carne roja & 240 & Ausencia & $<10$ & -- & -- & -- \\
\hline Norma & $<10$ & Ausencia & $<10$ UFC $/ \mathrm{ml}$ & -- & -- & -- \\
\hline Agua & $<1.1$ & Ausencia & -- & $<1.1$ & $<1.1$ & -- \\
\hline $\begin{array}{l}\text { Norma } \\
\text { Refresco }\end{array}$ & $<1.1 \mathrm{NMP} / 100 \mathrm{ml}$ & Ausencia & -- & $<1.1 \mathrm{NMP} / 100 \mathrm{ml}$ & $<1.1 \mathrm{NMP} / 100 \mathrm{ml}$ & 100 UFC/ml \\
\hline de mora & 4.6 & Ausencia & $<1$ & 4.6 & 11 & -- \\
\hline Norma & $<1.1 \mathrm{NMP} / 100 \mathrm{ml}$ & Ausencia & $<1.1 \mathrm{NMP} / 100 \mathrm{ml}$ & $<1.1 \mathrm{NMP} / 100 \mathrm{ml}$ & $<1.1 \mathrm{NMP} / 100 \mathrm{ml}$ & 100 UFC/ml \\
\hline
\end{tabular}

Nota: NMP: número más probable, UFC: unidades formadoras de colonias; P/A: Presencia/Ausencia, ausencia equivale < 3, y para Staphylococcus aureus < 10, g: gramos, ml: milímetros. 


\section{Evaluación de BPM a los manipuladores de alimentos}

En este instrumento se busca conocer tres variables sociodemográficas (edad, nivel de estudio y municipio de residencia). La parte siguiente es un cuestionario con algunas preguntas cerradas y otras son abiertas (dicotómicas y policotómicas). Con este tipo de preguntas se espera recolectar datos sobre las incidencias que se dan durante el proceso de elaboración de alimentos.

¿Ha recibido capacitación para manipulación de alimentos?

En esta pregunta, un $68 \%$ de los encuestados respondió que ha recibido capacitación; y el 32 \% restante dijo que no ha recibido capacitación en manipulación de alimentos (ver tabla 2).

Tabla 2

Capacitación en manipulación de alimentos

Capacitación en manipulación

de alimentos

\begin{tabular}{cc}
\multicolumn{3}{c}{ de alimentos } \\
\hline Sí & 17 \\
No & 8 \\
\hline
\end{tabular}

¿Cada cuánto recibe inspecciones por parte del Ministerio de Salud (Minsal)?

En esta pregunta la mitad de los encuestados opina que son visitados una vez al año. El $20 \%$ recibe visitas una vez al mes y el $28 \%$ dice que nunca lo han visitado del Minsal (ver tabla 3).

Tabla 3

Inspecciones del Ministerio de Salud hacia los locales

\begin{tabular}{lc}
\hline Tiempo & Número de inspecciones \\
\hline Nunca & 7 \\
Una vez al mes & 5 \\
Una vez al año & 13 \\
\hline
\end{tabular}

\section{Conclusiones}

- Las encuestas realizadas a los manipuladores de alimentos reflejan que algunos tienen poco o nada de conocimiento en el manejo de alimentos, hacen uso de utensilios para servir alimentos crudos y cocinados contaminando así otros alimentos.

- Uno de los hallazgos más relevantes en las encuestas es que el control de salud del manipulador en la mayoría de los locales no existe, siendo el manipulador el mayor contaminador de los alimentos debido a la manipulación de los alimentos.

- Para el agua de la red pública, sí cumple la norma salvadoreña obligatoria (NSO) 13.07.01:99, que establece los límites microbiológicos. En contraste, el refresco que se elabora a partir de la misma agua presenta, según análisis microbiológicos realizado, contaminación por coliformes fecales en el orden de 4.6, el número más probable por gramo de muestra; coliformes totales en el orden de 11, el número más probable por gramo (NMP/g) de muestra; y Escherichia coli en el orden de 4.6, el número más probable por gramo (NMP/g) de muestra. Todos estos son indicadores de contaminación que superan la norma salvadoreña obligatoria (NSO) 67.18.01:01 para bebidas no carbonatadas sin alcohol. No todos los locales utilizan agua de la red pública; algunos utilizan agua embotellada. Esto es evidencia de un proceso inadecuado en la elaboración del refresco, en el que la higiene de los manipuladores no es la adecuada a las buenas prácticas de manipulación de alimentos.

\section{Recomendaciones}

- Los cuchillos, tablas de cortar y recipientes de almacenamiento deben limpiarse y desinfectarse con regularidad y con la frecuencia suficiente para reducir al mínimo o evitar la contaminación.

- Se debe utilizar un desinfectante adecuado que tenga la capacidad de destruir el 99,99\% de las bacterias en el área de lavado de platos, servicios sanitarios, utensilios de cocina.

- Se debe verificar la salud de los manipuladores periódicamente. 
- Solo deben de utilizar materias primas seguras y utilizar métodos seguros de desinfección para las verduras.

- Utilizar ropa exclusivamente solo para el área de cocina y usar un jabón líquido antibacterial adecuado para el lavado de manos.

\section{Referencias bibliográficas}

Codex Alimentarius/Principios generales de higiene CAC/RCP 1 (1969). Recuperado de: http://www. codexalimentarius.org/standards/list-of-standards/

Codex Alimentarius CAC/RCP (2003). "Principios generales de higiene de los alimentos". Recuperado de: http:// www.codexalimentarius.org/standards/list-ofstandards/es/?provide=standards\&orderField=fullRef erence\&sort=asc\&num $1=\mathrm{CAC} / \mathrm{RCP}$

Código Alimentario Argentino (2013). "Buenas prácticas de manufactura". Resolución 80/96 del Reglamento. Reglamento del Mercosur. Recuperado de: http:// www.inan.gov.py/docs/80.96.pdf

Conacyt (2002). Norma salvadoreña Conacyt. NSO 67.18.01.01. "Productos Alimenticios. Bebidas no carbonatadas sin alcohol. Especificaciones". Recuperado de: http://www.defensoria.gob. sv/images/stories/varios/NORMAS/BEBIDAS/ ns067.18.01.01NO\%20CARBONATADAS $\% 20$ SIN\%20 ALCOHOL.pdf

FAO (2013). "Guía para muestreo de alimentos". Recuperado de: http://www.itp.gob.pe/normatividad/demos/doc/ Normas\%20Internacionales/Union\%20Europea/FAO/ GuMuestreoAlimento.pdf

FAO/OMS. (2003). "Principios generales de higiene en los alimentos". Recuperado de: http://www. codexalimentarius.org/standards/list-of-standards/es
/?provide=standards\&orderField=fullReference \&sort $=$ asc \&num $1=C A C / R C P$

Fundación Vasca para la seguridad alimentaria Elika (2013), "Manipulación de alimentos" (manual común). Recuperado de: http://www.juntadeandalucia.

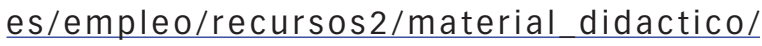
especialidades/materialdidactico manipulacion alimentos/PDF/Manual_Comun.pdf

Hernández, R., Fernández, C. \& Baptista, P. (2010). Metodología de la investigación. Quinta edición, McGraw-Hill. Disponible en:

http://www.mediafire.com/download/l1tyctzhau9y7ro/Met odologia+de+la+Investigacion+5ta+edicion.rar

OMS (2007). Manual sobre las cinco claves para la inocuidad de los alimentos. Departamento de inocuidad de los alimentos, zoonosis y enfermedades de transmisión alimentaria. Recuperado de: http://www.who.int/ foodsafety/publications/consumer/manual keys es.pdf

OPS Uruguay (2013). Enfermedades transmitidas por los alimentos. Recuperado de: http://www.bvsops.org. uy/pdf/etas.htm

OPS Uruguay (2013). Salmonella. Biblioteca virtual en salud. Recuperado de: http://www.bvsops.org.uy/pdf/ salmonella.pdf

Organización Panamericana de la Salud (2013). Manual de capacitación para manipuladores de alimentos). Recuperado de: http://www.soriano.gub.uy/www/ manual_manipuladores.pdf

Red Tercer Milenio (2012). "Métodos de conservación de alimentos". Recuperado de: $\quad$ http://www.aliatuniversidades.com. $\mathrm{mx} /$ bibliotecasdigitales/pdf/economico administrativo/M\%C3\%A9todos de conservacion_de_alimentos.pdf 